\title{
Steady current induced vibration of near-bed piggyback pipelines: Configuration effects on VIV suppression
}

\author{
Zhi-Peng Zang, Fu-Ping Gao* \\ Key Laboratory for Mechanics in Fluid Solid Coupling Systems, Institute of Mechanics, Chinese Academy of Sciences, Beijing 100190, China
}

\section{A R T I C L E I N F O}

\section{Article history:}

Received 22 June 2013

Received in revised form 12 February 2014

Accepted 17 February 2014

Available online 15 March 2014

\section{Keywords:}

Piggyback pipelines

Vortex induced vibration

Steady currents

VIV suppression

\begin{abstract}
A B S T R A C T
A series of experiments on steady current induced vibration of piggyback pipelines close to a plane seabed were conducted with a hydro-elastic facility in a conventional water flume. The effects of the mass-damping parameter, the diameter ratio, the gap-to-diameter ratio, the spacing-to-diameter ratio and the position angle on the VIV response were studied. The VIV suppression for the piggyback pipeline system by the small pipe was investigated based on the analysis of the vibration amplitude and the critical reduced velocity for the onset of VIV. Comparison with the prediction with the modified Griffin plot by Govardhan and Williamson (2006) [10] shows that the peak vibration amplitude of near-wall piggyback pipelines is smaller than that for a wall-free single pipe. The configuration parameters of piggyback pipelines have significant effects on the VIV suppression. For the configuration of the small pipe above the main pipe $\left(\theta=90^{\circ}\right)$, the minimum peak amplitude and the maximum critical reduced velocity occur at the spacing-to-diameter ratio of $G / D \approx 0.25$, indicating that VIV is suppressed most effectively by the small pipe at this value of $G / D$. For a constant value of $G / D=0.25$, both the minimum peak amplitude and the maximum critical reduced velocity occur at the position angle of $\theta \approx 120^{\circ}$.
\end{abstract}

(C) 2014 Elsevier Ltd. All rights reserved.

\section{Introduction}

Submarine pipelines are widely used in the offshore oil and gas engineering for transporting oil and gas products. For technical and economic reasons, a secondary (small) pipe is often bound to the primary (main) pipeline, forming the so-called "piggyback pipelines". The flow around the piggyback pipelines is more complex than that around a single pipeline. The vortex shedding from the pipeline system in flows could be suppressed due to the existence of the small pipe $[1,2]$, which may consequently suppress the vortex-induced vibration (VIV) of the piggyback pipelines with certain configurations.

Elastically-mounted systems in wind tunnels or water flumes are very efficient facilities for studies on VIV responses and the corresponding vortex shedding modes of a cylinder. One of the most famous experiments was conducted by Feng [3]. In his work, the mass ratio is very high $(\gg 1)$ as the experiments were conducted in the air. The oscillation frequency was found to be very close to the natural frequency of the structure in the lock-in regime. However, for the cases of lower mass ratios (e.g. in the water), the oscillation frequency does not follow the natural frequency and shows a compromise between the natural frequency and the Strouhal frequency $[4,5]$. Khalak and Williamson [5] found that the response regime of the reduced velocity at low mass ratios is wider than that at high mass ratios. Some reviews on the topic of VIV can be referenced in [6-9].

The combined mass-damping parameter has been an important parameter in predicting the maximum amplitude of VIV over the last three decades, although there was still a large scatter in peak-amplitude versus the mass-damping parameter in the Griffin plot [9]. Govardhan and Williamson [10] conducted a systematic study on the effects of system mass and damping on the peak vibration amplitude with an accurately controlled-damping facility. Their work indicated that the combined mass-damping parameter does collapse peak-amplitude data very well

\footnotetext{
* Corresponding author. Tel.: +86 10 82544189; fax: +861062561284.

E-mail address: fpgao@imech.ac.cn (F.-P. Gao).
}

for a given Reynolds number and Reynolds number has an important influence on the value of peak amplitude. Generally, the peak amplitude increases with the increase of the Reynolds number for a given mass-damping parameter. In Klamo et al. [11], it was found that because of the influence of Reynolds number, the traditional labels of "high mass-damping" and "low mass-damping" are incomplete with regard to predicting a large or small-amplitude response profile. Vandiver [12 recently gave a brief historical review of mass-damping from 1955 to 2006, and proposed an updated dimensionless damping parameter well-suited for predicting the VIV response of flexible risers in sheared flows.

There were also many studies focused on VIV of two-cylinder system. The vibration amplitude of two cylinders depends on their relative locations and the vibration of one cylinder can affect the vortex shedding and synchronization of the other $[13,14]$. For free vibrations of two side-by-side cylinders in a cross-flow, the system modal damping ratio and the natural frequency were found to be dependent on the spacing ratio [15]. Assi et al. [16] investigated the mechanism of wake-induced vibrations (WIV) of a pair of cylinders in a tandem arrangement. It was indicated that, different from the typical VIV response, the WIV response was characterized by a build-up of amplitude persisting to high reduced velocities.

The piggyback pipelines are a special system of two cylinders typically with different diameters. Some existing investigations shown that, the existence of the small pipe can bring a pronounced increase in the drag force upon the main pipe and the maximum value of the mean drag force occurs in the side-by-side arrangement [17]. The fluctuation of the lift coefficient is weak when the two cylinders are in a tandem arrangement and strong in a side-by-side arrangement $[18,19]$. When the piggyback pipes are in the proximity of a plane boundary (e.g. the seabed), both the configuration and boundary effects are obvious on the suppression of the vortex shedding from the structure $[1,2]$.

Till now, there exist few studies on the VIV response of piggyback pipelines. Zang et al. [2] measured the wake flow field around near-bed piggyback pipelines with the PIV technique. The suppression of vortex shedding from the main pipe was investigated with the swirling strength analysis and the result was validated with the amplitude of transverse VIV at the reduced velocity of 6.0. In Rahmanian et al. [20], the influence of the position of the small cylinder on the VIV response was 
studied at the reduced velocity of 8.0. Zhou and Lalli [21] conducted experiments in the air at a mass ratio of about 245 . It was found that the largest amplitude occurs at $\theta=90^{\circ}$ and the smallest value occurs at $\theta=135^{\circ}$. The amplitude of VIV decreases with the increase of the spacing ratio for most of configurations, accompanied by a decreasing reduced velocity for peak amplitudes.

As aforementioned, the existing studies on the VIV of piggyback pipelines focused either on certain values of the reduced velocity or in air conditions with high mass ratios, the overall VIV responses of piggyback pipelines for the typical range of the reduced velocity under the field conditions have not been investigated comprehensively. Det Norske Veritas (DNV) RP-F105 [22] similarly suggested that, particularly for VIV of special pipeline designs, including piggyback pipelines, bundled pipelines, with limited experience, experiments should be considered.

In this study, the transverse VIV of piggyback pipelines close to a plane boundary in a steady flow was physically modeled with a hydro-elastic facility in a large water flume. Based on dimensional analyses, a series of tests have been conducted to examine the configuration effects on VIV suppression for the piggyback pipelines.

\section{Dimensional analyses and experimental details}

\subsection{Dimensional analyses}

The VIV responses of submarine piggyback pipelines under the action of a steady current involve complex interactions between the current, pipelines and the seabed. Based on dimensional analyses, the vibration amplitude ratio $(A / D)$, the vibration frequency ratio $\left(f / f_{n}\right)$, the critical reduced velocity for the onset of $\operatorname{VIV}\left(V_{r, \text { onset }}\right)$ and the force coefficients $\left(C_{D}\right.$ and $\left.C_{L}\right)$ are mainly relative to the following non-dimensional groups, respectively:

$$
\begin{aligned}
& A / D=\lambda_{1}\left(e / D, G / D, d / D, \theta, V_{r}, \operatorname{Re}, \alpha, \ldots\right) \\
& f / f_{n}=\lambda_{2}\left(e / D, G / D, d / D, \theta, V_{r}, \operatorname{Re}, \alpha, \ldots\right) \\
& V_{r, \text { onset }}=\lambda_{3}(e / D, G / D, d / D, \theta, \operatorname{Re}, \alpha, \ldots) \\
& C_{D}=\lambda_{4}\left(e / D, G / D, d / D, \theta, V_{r}, \operatorname{Re}, \alpha, \ldots\right) \\
& C_{L}=\lambda_{5}\left(e / D, G / D, d / D, \theta, V_{r}, \operatorname{Re}, \alpha, \ldots\right)
\end{aligned}
$$

where $A$ is the vibration amplitude, i.e. $A=\left(y_{\max }-y_{\min }\right) / 2$, in which $y_{\max }$ and $y_{\min }$ are the maximum and the minimum displacement of the pipe system in the transverse $y$-direction (perpendicular to the free stream), respectively; $D$ is the outer diameter of the main pipe; $e$ is the gap between the main pipe and the bottom, and $e / D$ denotes the gap-to-diameter ratio; $G$ is the spacing between two pipes, and $G / D$ denotes the spacing-to-diameter ratio; $d$ is the outer diameter of the piggyback (small) pipe, and $d / D$ denotes the diameter ratio; $\theta$ is the position angle of the piggyback pipe relative to the main pipe; $f$ is the oscillation frequency, $f_{n}$ is the natural frequency of the system of piggyback pipes in the still water. The reduced velocity $V_{r}$ is defined as

$V_{r}=\frac{U}{D f_{n}}$

where $U$ is the free-stream velocity. $\alpha$ is the combined massdamping parameter, i.e.

$\alpha=\left(m^{*}+C_{a}\right) \zeta$

in which the mass ratio $m^{*}$ of the piggyback pipes is defined as

$m^{*}=\frac{4 m}{\pi \rho\left(D^{2}+d^{2}\right)}$

where $m$ is the total mass of the oscillating structure per unit length and $\rho$ is the mass density of the water; $C_{a}$ is the potential flow added mass coefficient ( $C_{a}=1.0$ for a circular cylinder); $\zeta$ is the damping factor of the system, the method for determining of which is given in Section 2.2. $R e(=U D / v)$ is the Reynolds number of the main pipe, where $v$ is the kinematic viscosity of the water.

The critical reduced velocity $\left(V_{r, \text { onset }}\right)$ for the onset of VIV is a crucial parameter for evaluating the allowable length to avoid VIV of the spanning pipeline. In the DNV RP-F105 [22], the referenced value for $V_{r, \text { onset }}$ is set as the one at which $A / D$ is increased

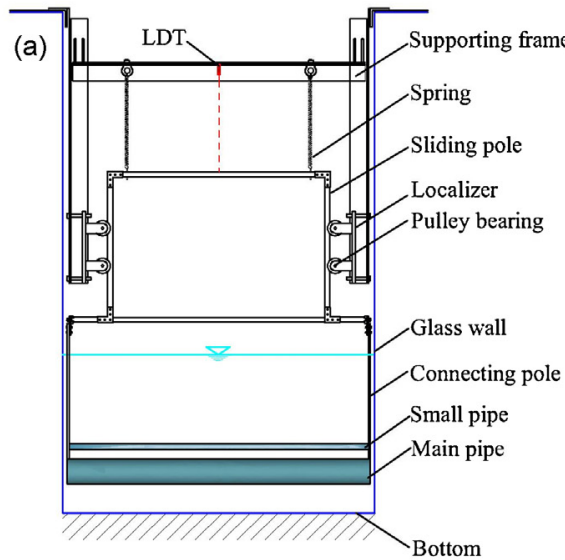

Front view

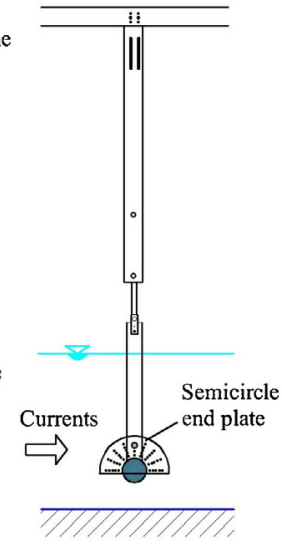

Side view (b)

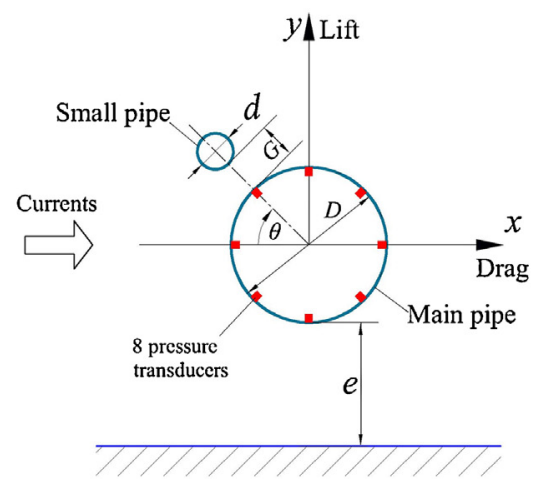

Fig. 1. (a) Details of VIV modeling system and (b) configuration of piggyback pipelines and arrangement of pressure transducers.

(from nil) up to 0.15 while increasing the flow velocity, i.e. $V_{r, \text { onset }}=$ $\left.V_{r}\right|_{A / D=0.15}$. In this study, the same criterion is adopted for the critical reduced velocity. The in-line drag force coefficient $C_{D}$ and the transverse lift force coefficient $C_{L}$ are expressed as follows:

$C_{D}=\frac{F_{D}}{0.5 \rho D U^{2}}$

$C_{L}=\frac{F_{L}}{0.5 \rho D U^{2}}$

where $F_{D}$ and $F_{L}$ are the total in-line and transverse force per unit length on the main pipe, respectively. The details for the measurement method of these hydrodynamic forces are given in Section 2.2 .

\subsection{Experimental details}

A hydro-elastic facility in conjunction with a water flume was employed for physical modeling VIV responses of the piggyback pipes (see Fig. 1). The water flume $(52.0 \mathrm{~m}$ long, $1.0 \mathrm{~m}$ wide and $1.5 \mathrm{~m}$ deep) located at the Institute of Mechanics, Chinese Academy of Sciences, is capable of generating steady flow with velocity up to approximately $0.75 \mathrm{~m} / \mathrm{s}$ with the water depth set at $0.5 \mathrm{~m}$. The hydro-elastic facility is updated from the one ever used by Gao et al. [23]. The model pipes were attached to the supporting frame by two connecting poles, two sliding poles and two sets of springs. The sliding poles can move along the four pulley bearings, which are connected to the two localizers. In the present study, only transverse vibration was simulated with the hydro-elastic facility. 
The model of the main pipe is made of a plexiglass tube $(D=80 \mathrm{~mm})$. Four small pipe models made of a plexiglass rod with various diameters, i.e. $d=12 \mathrm{~mm}, 20 \mathrm{~mm}, 30 \mathrm{~mm}$ and $40 \mathrm{~mm}$ were used, respectively. The length of the piggyback pipes is $980 \mathrm{~mm}$. As shown in Fig. 1(a), a pair of semicircle-shaped plates was utilized at the both ends to position the two model pipes, so that the position angle can be varied in the range of $\theta=0-180^{\circ}$ and the spacing-todiameter ratio $G / D=0-0.5$ (see Fig. 1 (b)). The mass ratio $m^{*}$ can be varied by adjusting the weight of the main pipe.

The oscillation of the piggyback pipes was measured with a laser displacement transducer (LDT) mounted on the supporting frame (see Fig. 1(a)). The natural frequency of the system of piggyback pipes $f_{n}$ was obtained by spectrum analyses of free-decay tests in the still water. The damping factor $\zeta$ was determined with the conventional free-decay testing method, i.e. applying a given excitation to the structure, then recording the vertical oscillation with the LDT. For slightly damped structures, the damping factor can be estimated with $\zeta=\ln \left(A_{i} / A_{i+n}\right) /(2 \pi n)$, where $A_{i}$ is the initial amplitude of pipe vibrations, $A_{i+n}$ is the amplitude after $n$ cycles. Note that the measured damping factor includes both structural and hydrodynamic sources.

An acoustic Doppler velocimetry (ADV) was placed at $3.0 \mathrm{~m}$ upstream the pipes and $0.12 \mathrm{~m}$ above the flume bottom to measure the flow velocity (see Fig. 1(b)). The tests were conducted by increasing the flow velocity gradually to reach steady states. For the maximum value of the flow velocity $(U \approx 0.75 \mathrm{~m} / \mathrm{s})$, the corresponding reduced velocity reached $V_{r} \approx 16$ and the Reynolds number reached $R e \approx 6.0 \times 10^{4}$.

The hydrodynamic forces on the main pipe were measured with 8 miniature pressure transducers, which were evenly distributed along the circumference of the main pipe's surface (see Fig. 1(b)). The hydrodynamic force on a bluff body in flows includes two components, i.e. the force due to pressure and the force due to friction. According to Achenbach [24], the contribution of the friction is usually insignificant $(<3 \%)$ compared with the contribution of the pressure for Reynolds numbers greater than $10^{4}$. In this study, the total hydrodynamic forces on the main pipe per unit length $\left(F_{D}\right.$ and $\left.F_{L}\right)$ are calculated by integrating the pressure along the circumference of the cylinder:

$F_{D} \approx \sum_{i=1}^{8} \frac{\pi}{8} D\left(p_{i}-p_{i 0}\right) \cos \alpha_{i}$

$F_{L} \approx-\sum_{i=1}^{8} \frac{\pi}{8} D\left(p_{i}-p_{i 0}\right) \sin \alpha_{i}$

where $p_{i}$ and $p_{i 0}$ denote the total transient pressure and the static pressure measured by the $i$ th pressure transducer, respectively; $\alpha_{i}$ is the position angle of the $i$ th transducer. Submitting the values of $F_{D}$ and $F_{L}$ into (5a) and (5b), the drag force coefficient $C_{D}$ and the lift force coefficient $C_{L}$ can be obtained.

Parametric study involving 23 tests in total has been made to reveal the mechanism of VIV of the piggyback pipelines. The test conditions and the main results for VIV responses of the piggyback pipes are listed in Table 1.

\section{Results and discussion}

3.1. Effect of mass-damping and Reynolds number: comparison with 'modified Griffin plot' [10]

The effect of the mass-damping parameter and Reynolds number on the VIV responses of piggyback pipes is investigated, including the critical reduced velocity for the onset of VIV, peak amplitude of VIV, etc. Three values of the mass-damping parameters are chosen, i.e. $\alpha=0.145,0.161$ and 0.182 , with the Reynolds number of the maximum VIV amplitude $\left.R e\right|_{(A / D)_{\max }}=3.8 \times 10^{4}, 3.2 \times 10^{4}$, and $2.5 \times 10^{4}$, respectively (see Table 1 ).
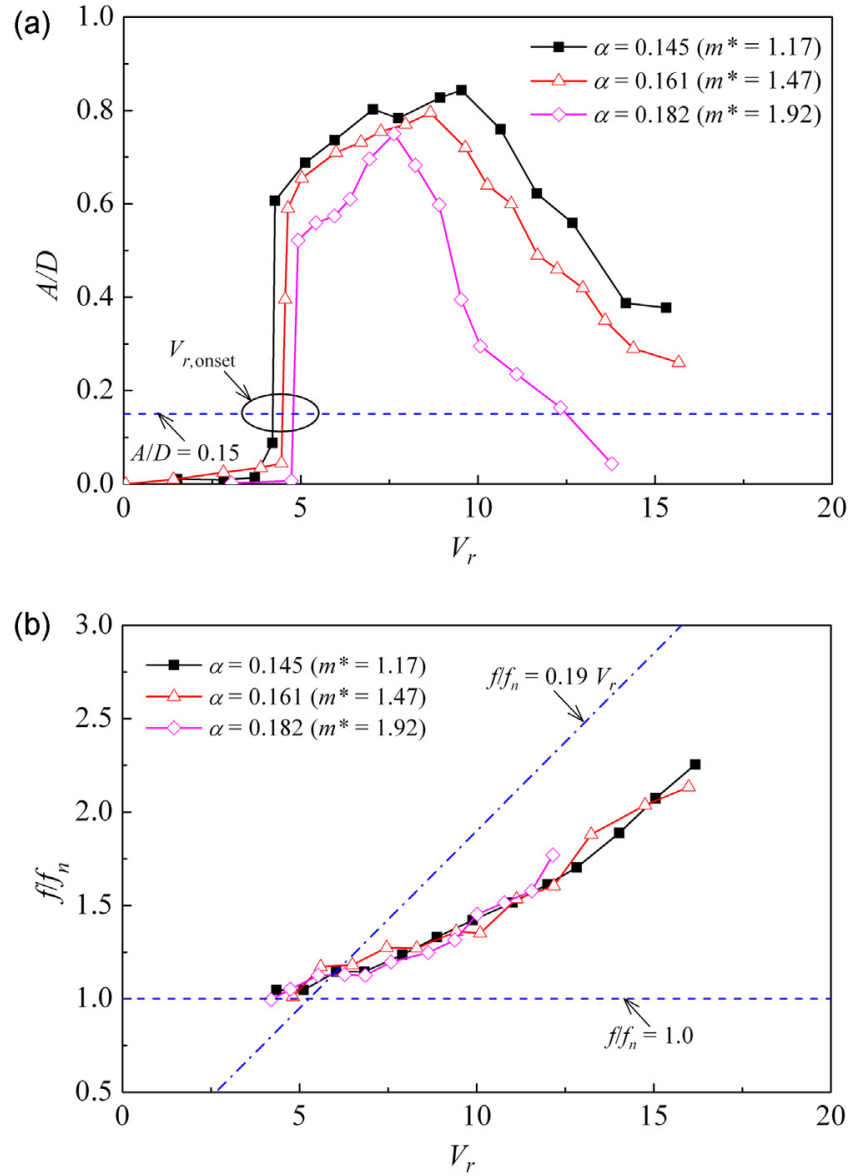

Fig. 2. VIV response of piggyback pipelines for different mass-damping parameters: (a) amplitude and (b) frequency $\left(e / D=1.0, G / D=0.25, d / D=0.25, \theta=90^{\circ}\right.$, $\left.R e=2.5-3.8 \times 10^{4}\right)$.

As aforementioned in Section 2.1, the critical reduced velocity $\left(V_{r, \text { onset }}\right)$ is one of the most important parameters for the evaluation of VIV suppression. In Fig. 2(a), the line of $A / D=0.15$ is provided to obtain the value of $V_{r, \text { onset }}$. It can be seen that $V_{r, \text { onset }}$ increases with the increase of $\alpha$, indicating that increasing the mass-damping parameter may delay the onset of VIV, which reflects the role of the system massdamping in the VIV suppression. Moreover, the width of the lock-in range also decreases with the increase of the mass damping parameter, which is coincident with the previous observations for a single cylinder (e.g. $[5,26])$.

The normalized frequency of VIV is plotted against $V_{r}$ in Fig. 2(b). Lei et al. [25] indicated that the Strouhal number of the vortex shedding from a circular cylinder close to a plane boundary is about 0.19 at the gap-to-diameter ratio of $e / D=1.0$ if the boundary layer thickness is greater than the cylinder diameter. Both the lines of $f / f_{n}=0.19 V_{r}$ and $f / f_{n}=1.0$ are plotted on Fig. 2(b) for comparisons. The vibration frequency shows a compromise between the natural frequency and the Strouhal frequency. $f / f_{n}$ increases with the increase of $V_{r}$. The effect of the mass-damping parameter on the response frequency is not obvious for these test cases.

As illustrated in Fig. 2(a), the value of $A / D$ decreases with the increasing $\alpha$ in the lock-in range. The peak amplitude $(A / D)_{\max }$ of the VIV of piggyback pipes are plotted against $\alpha$ in a modified Griffin plot [10], in which the influence of Reynolds number is taken into account (see Fig. 3). In Govardhan and Williamson [10], an empirical formula was proposed to predict the peak amplitude of VIV for a wall-free single cylinder:

$(A / D)_{\max }=\left(1-1.12 \alpha+0.30 \alpha^{2}\right) \lg \left(0.41\left(\left.\operatorname{Re}\right|_{(A / D)_{\max }}\right)^{0.36}\right)$

Generally, in the modified Griffin plot, $(A / D)_{\max }$ decreases with the increase of $\alpha$ for a fixed value of $\left.R e\right|_{(A / D)_{\max }}$ and increases with the increase of $\left.R e\right|_{(A / D)_{\max }}$ for a fixed value of $\alpha$. In Fig. 3, the predicted values by Eq. (7) are plotted at the corresponding Reynolds numbers of the peak amplitudes of present tests, i.e. $\left.\operatorname{Re}\right|_{(A / D)_{\max }}=2.5 \times 10^{4}, 3.2 \times 10^{4}$ and $3.8 \times 10^{4}$. It is indicated that the present test results for the piggyback pipes follow the same trends as predicted with the modified Griffin plot for the wall-free singe cylinder, with smaller values by about $25 \%$. For the near-bed piggyback pipes, the presences of the secondary (small) pipe and the boundary have obvious effect on the vibration suppression resulting from the suppression of vortex shedding as observed in [2]. 
Table 1

Test conditions and results for VIV of piggyback pipelines.

\begin{tabular}{|c|c|c|c|c|c|c|c|c|c|c|c|c|}
\hline \multirow[t]{2}{*}{ Test no. } & \multicolumn{8}{|c|}{ Test conditions } & \multicolumn{3}{|c|}{ Test results } & \multirow[t]{2}{*}{ Section } \\
\hline & $d / D$ & $e / D$ & $G / D$ & $\theta\left({ }^{\circ}\right)$ & $m^{*}$ & $f_{n}(\mathrm{~Hz})$ & $\zeta$ & $\alpha$ & $(A / D)_{\max }$ & $V_{r, \text { onset }}$ & $\left.\operatorname{Re}\right|_{(A / D)_{\max }}\left(\times 10^{4}\right)$ & \\
\hline 1 & 0.25 & 1.0 & 0.25 & 90 & 1.17 & 0.62 & 0.067 & 0.145 & 0.84 & 4.2 & 3.8 & 3.1 \\
\hline 2 & - & - & - & - & 1.47 & 0.59 & 0.065 & 0.161 & 0.80 & 4.5 & 3.2 & \\
\hline 3 & - & - & - & - & 1.92 & 0.52 & 0.062 & 0.182 & 0.76 & 4.9 & 2.5 & \\
\hline 4 & 0.15 & 1.0 & 0.25 & 90 & 1.47 & 0.60 & 0.066 & 0.162 & 0.82 & 4.1 & 2.6 & 3.2 .1 \\
\hline 5 & 0.38 & - & - & - & - & 0.58 & 0.064 & 0.158 & 0.76 & 5.7 & 3.3 & \\
\hline 6 & 0.50 & - & - & - & - & 0.56 & 0.062 & 0.153 & 0.73 & 7.2 & 2.6 & \\
\hline 7 & 0.25 & 0.1 & 0.25 & 90 & 1.47 & 0.62 & 0.064 & 0.158 & 0.62 & 10.2 & 4.2 & 3.2 .2 \\
\hline 8 & - & 0.3 & - & - & - & 0.60 & 0.063 & 0.155 & 0.70 & 9.6 & 3.7 & \\
\hline 9 & - & 0.5 & - & - & - & 0.58 & 0.061 & 0.151 & 0.78 & 5.2 & 3.2 & \\
\hline 10 & 0.25 & 1.0 & 0 & 90 & 1.47 & 0.58 & 0.067 & 0.165 & 0.97 & 3.9 & 2.6 & 3.2 .3 \\
\hline 11 & - & - & 0.13 & - & - & 0.58 & 0.065 & 0.161 & 0.88 & 4.1 & 2.1 & \\
\hline 12 & - & - & 0.19 & - & - & 0.58 & 0.065 & 0.161 & 0.85 & 4.3 & 2.3 & \\
\hline 13 & - & - & 0.31 & - & - & 0.58 & 0.062 & 0.153 & 0.81 & 4.3 & 2.6 & \\
\hline 14 & - & - & 0.38 & - & - & 0.58 & 0.064 & 0.158 & 0.81 & 4.2 & 2.3 & \\
\hline 15 & - & - & 0.50 & - & - & 0.58 & 0.063 & 0.155 & 0.81 & 4.1 & 2.5 & \\
\hline 16 & 1 & - & $(\infty)$ & 1 & - & 0.58 & 0.066 & 0.164 & 0.82 & 4.0 & 2.2 & (single) \\
\hline 17 & 0.25 & 1.0 & 0.25 & 0 & 1.47 & 0.57 & 0.066 & 0.163 & 0.92 & 3.2 & 4.6 & 3.2 .4 \\
\hline 18 & - & - & - & 30 & - & 0.57 & 0.065 & 0.161 & 0.57 & 3.8 & 1.8 & \\
\hline 19 & - & - & - & 45 & - & 0.57 & 0.064 & 0.158 & 0.65 & 3.5 & 2.3 & \\
\hline 20 & - & - & - & 60 & - & 0.57 & 0.065 & 0.161 & 0.95 & 3.7 & 2.2 & \\
\hline 21 & - & - & - & 120 & - & 0.57 & 0.066 & 0.163 & 0.50 & 5.4 & 2.2 & \\
\hline 22 & - & - & - & 150 & - & 0.57 & 0.066 & 0.163 & 0.65 & 5.1 & 1.9 & \\
\hline 23 & - & - & - & 180 & - & 0.57 & 0.065 & 0.161 & 0.72 & 3.9 & 2.6 & \\
\hline
\end{tabular}

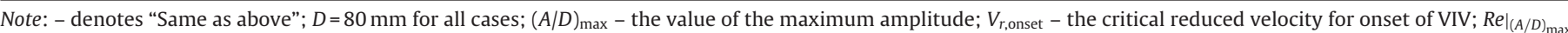
- the value of Reynolds number for occurrence of the maximum VIV amplitude.

\subsection{Effect of configuration parameters}

\subsubsection{Effect of diameter ratio $d / D$}

The effect of the diameter ratio on the VIV response of piggyback pipelines is examined at four values of the diameter ratio, i.e. $d / D=0.15,0.25,0.375$ and 0.5 . As the specific gravity of the plexiglass $(\sim 1.18)$ is very close that of the water, the increase in the diameter of the small pipe has slight effect on the mass ratio of the whole system $(<5 \%)$. The mass ratio is kept at $m^{*} \approx 1.47$.

Fig. 4(a) gives the variations of the vibration amplitude with the reduced velocity. The right boundary of the lock-in regime is greater than the maximum reduced velocity that the water flume can generate. Judged by the trends of the curves, it appears that the right boundary reduced velocity of the lock-in regime at $d / D=0.15$ is the smallest and that at $d / D=0.5$ is the largest.

The critical reduced velocity $V_{r \text {,onset }}$ increases with the increase of $d / D$, indicating that increasing the diameter of the small pipe may delay the onset of VIV. The peak amplitude of VIV also decreases with the increases of the diameter. The main reason for the decreasing amplitude is that an increase in the small pipe diameter effectively weakens the vortex shedding from the main pipe [2]. The effect of Reynolds number on the peak amplitude would be very small as $\left.R e\right|_{(A / D \max }$ varies in a narrow range $\left(2.6 \times 10^{4}-3.3 \times 10^{4}\right)$ in these cases.

In Fig. 4(b), the frequency of VIV increases with the increase of the reduced velocity. The effect of the diameter ratio on the frequency is weak as $V_{r}<10$. At $V_{r}>10$, the response frequency decreases with the increase of $d / D$.

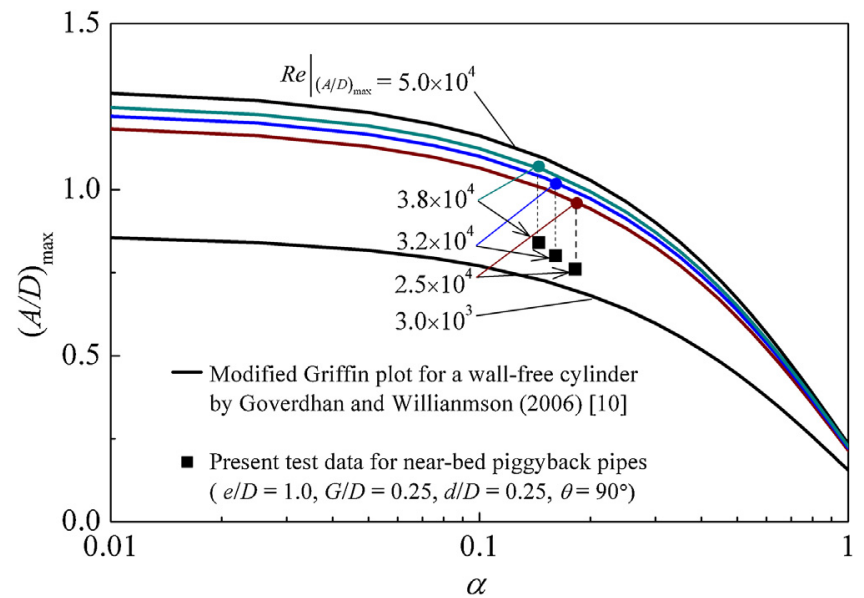

Fig. 3. Comparisons between peak amplitudes for near-wall piggyback pipelines and predicted values for a wall-free cylinder by modified Griffin plot.
3.2.2. Effect of gap-to-diameter ratio e/D

The near-wall effect on VIV of piggyback pipelines is studied, by conducting the tests at $e / D=0.1,0.3,0.5$ and 1.0 , respectively. The small pipe was placed above the main pipe $\left(\theta=90^{\circ}\right)$ with a spacing-to-diameter ratio of $G / D=0.25$.
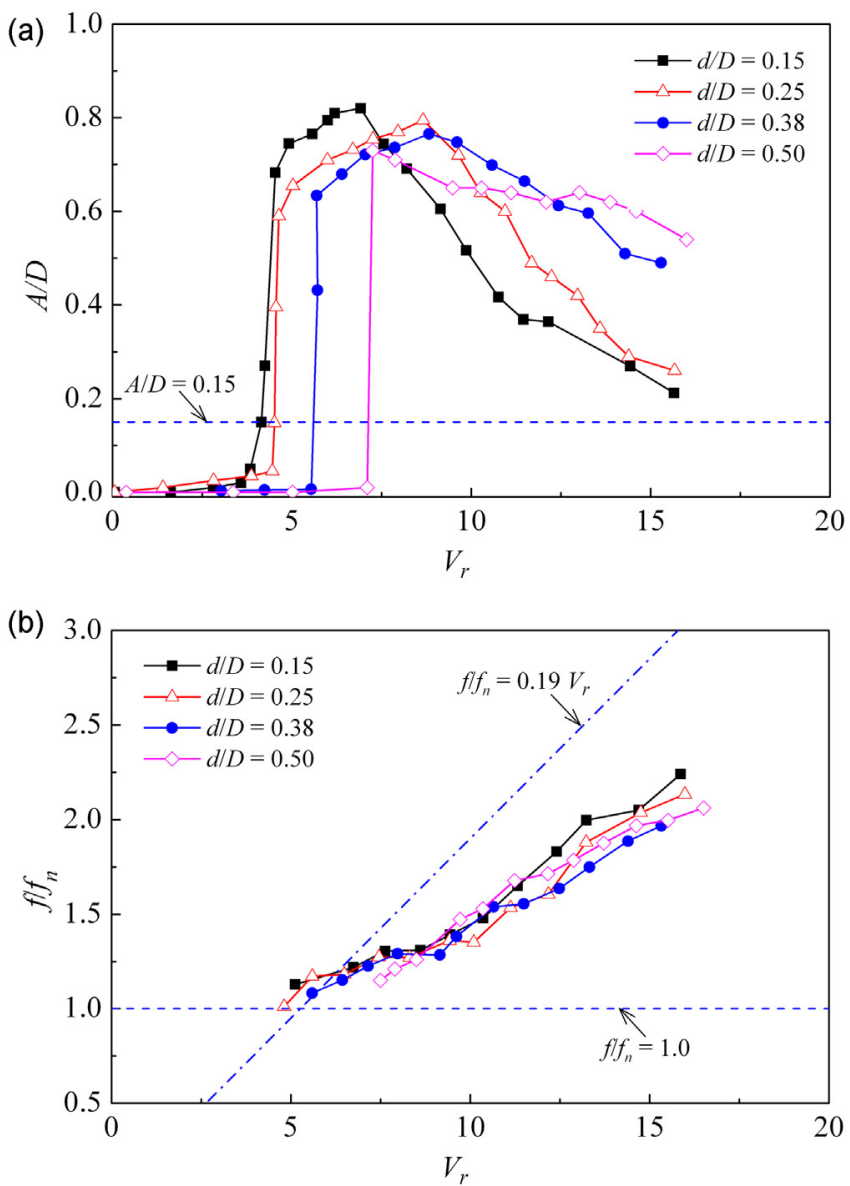

Fig. 4. VIV response of piggyback pipelines for different diameter ratios: (a) amplitude and (b) frequency $\left(e / D=1.0, G / D=0.25, \theta=90^{\circ}, \alpha \approx 0.160, R e=2.6-3.3 \times 10^{4}\right)$. 


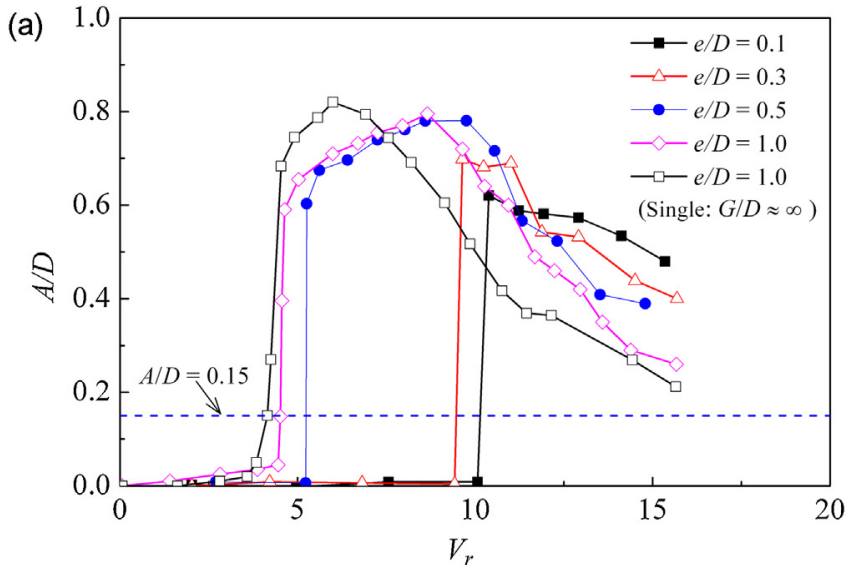

(b)

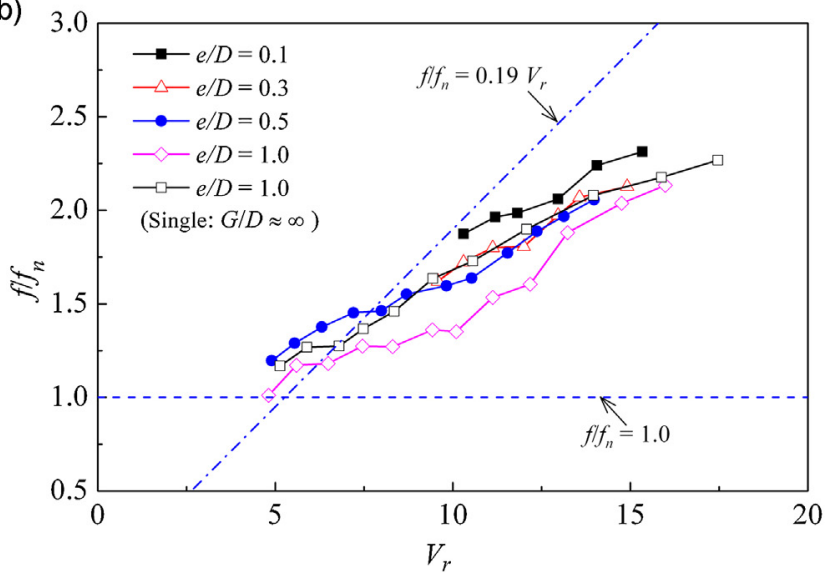

Fig. 5. VIV response of piggyback pipelines for different gap-to-diameter ratios: (a) amplitude and (b) frequency $\left(G / D=1.0, d / D=0.25, \theta=90^{\circ}, \alpha \approx 0.156\right.$, $\left.R e=3.2-4.2 \times 10^{4}\right)$.

The diameter ratio is set at $d / D=0.25$ and the mass-damping parameter is $\alpha \approx 0.156$.

In Fig. 5(a), it can be seen that the peak amplitude of VIV generally decreases with the decreasing $e / D$ in the lock-in regime for near-wall piggyback pipelines, even though the Reynolds number of the peak amplitude is increasing. The same trend has been found for a near-wall single pipe by Yang et al. [26]. This can be related to the vortex shedding suppression on the main pipe by the bottom [2]. The amplitude of VIV at $e / D=1.0$ is slightly larger than that at $e / D=0.5$, which indicates that the effect of the bottom would be ignored if $e / D$ is large enough. The data for VIV of a single pipe at $e / D=1.0$ is also shown in the figure for comparison. At the same gapto-diameter ratio $e / D=1.0$, the maximum vibration amplitude of piggyback pipeline is smaller and the critical reduced velocity is larger than that of a single pipe, which indicates that the existence of the small pipe have much effect on VIV suppression.

The critical reduced velocity $V_{r \text {,onset }}$ increases with the decrease of $e / D$. The value of $V_{r \text {,onset }}$ at $e / D=0.1$ is about 10 , which is much larger than the value for a single pipe $\left(V_{r, \text { onset }}=6\right.$ ) at the same value of $e / D[26]$. This is mainly due to that the existence of the small pipe also contributes to the suppression of the vortex shedding, in addition to the effect of the bottom.

The normalized frequency of VIV is plotted against $V_{r}$ in Fig. 5(b). $f / f_{n}$ with the increase of $V_{r}$ for all the tested gap-to-diameter ratios. The normalized frequency of VIV for piggyback pipelines decreases with the increase of $e / D$, which is similar to the trend for a single pipeline close to a plane boundary [26].

\subsubsection{Effect of spacing-to-diameter ratio $G / D$}

The effect of the spacing-to-diameter ratio on the VIV response of piggyback pipelines is studied with $G / D$ ranging in $0 \sim 0.5$. The position angle is set at $\theta=90^{\circ}$. The diameter ratio is $d / D=0.25$. The mass-damping parameter is $\alpha \approx 0.160$.

In Fig. 6(a), the vibration amplitudes of the piggyback pipelines are plotted against the reduced velocity. It can be seen that the vibration amplitude and the width of the lock-in range are very dependent on $G / D$. With the increase of the flow velocity (from nil), the amplitude of VIV jumps suddenly to a large value at the critical reduced velocity. For $G / D=0$ and 0.13 , the width of the lock-in range is larger than that for other cases and the right boundary of the lock-in regime is beyond the maximum reduced velocity that the facility can generate. There is slight difference

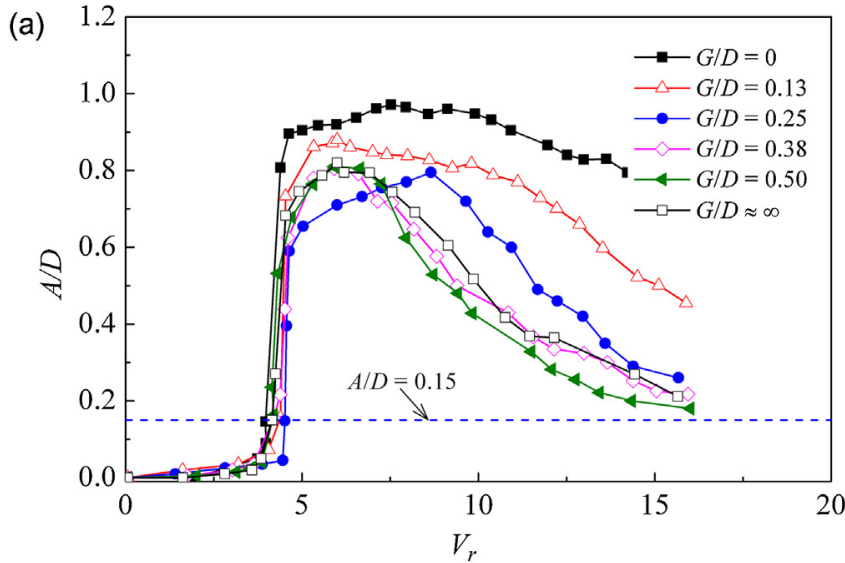

(b)

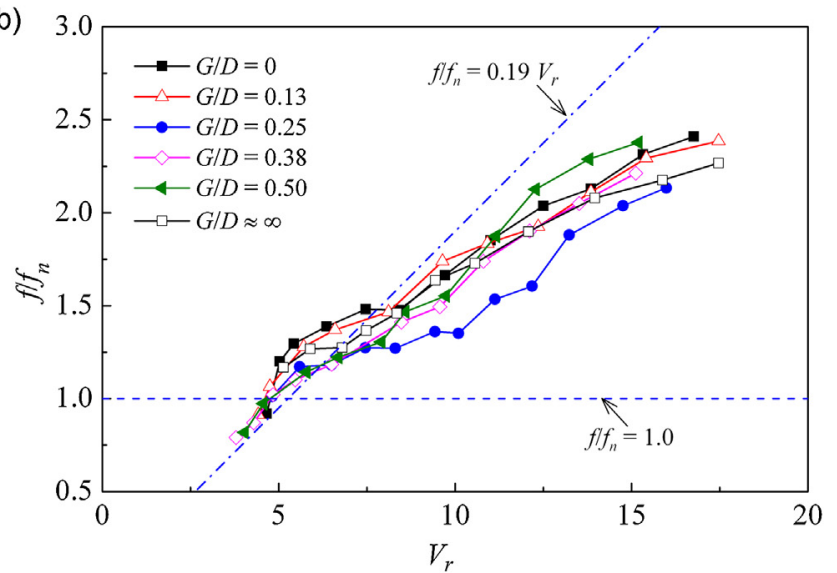

Fig. 6. VIV response of piggyback pipelines for different spacing-to-diameter ratios: (a) amplitude and (b) frequency $\left(e / D=1.0, d / D=0.25, \theta=90^{\circ}, \alpha \approx 0.160\right.$, $\left.R e=2.1-3.2 \times 10^{4}\right)$.

in the vibration amplitude and the width of the lock-in range among $G / D=0.38,0.50$ and infinite (i.e. single pipe).

In Fig. $6(\mathrm{~b})$, the normalized frequencies $\left(f / f_{n}\right)$ generally increases with the increase of $V_{r}$. When the reduced velocity is small $\left(V_{r}<6\right), f / f_{n}$ basically follows the Strouhal line, indicating that vortex shedding dominates the vibration of the piggyback pipeline. $f / f_{n}$ is slightly greater than 1.0 and increases very slowly with the increasing $V_{r}$ at $6<V_{r}<9$. It is likely that the response frequency lock-in with the vortex shedding frequency in the range of $6<V_{r}<9$, resulting in the maximum amplitude as shown in Fig. 6(a).

Fig. 7(a) shows the normalized mean position of the vibration varying with the increasing reduced velocity, where the normalized mean position is defined as $\left(y_{\max }+y_{\min }\right) / 2 D$. The mean position of the vibration is not a linear function of $G / D$. For a single pipe $(G / D \approx \infty)$, the mean position is very close to the initial position (zero) due to the symmetric configuration. The slight upward deflection likely stems from the effect of the bottom. As for $G / D=0$, the two pipes are in contact with each other and can be approximated by a single pipe with a larger dimension in the cross-flow direction. The little difference between the mean position at $G / D=0$ and $G / D \approx \infty$ is due to the existence of the small pipe. For $G / D=0.5$, the two pipes are far separated from each other and the interference between their wake flows is insignificant. Thus, the mean position is also small and close to the value for a single pipe. The highest mean position occurs at a medium spacing of $G / D=0.25$.

The mean drag and lift coefficients on the main pipe at fixed reduced velocity of $V_{r}=9\left(R e=3.4 \times 10^{4}\right)$ are shown in Fig. 7(b). It can be seen that the mean value of $C_{D}$ decreases with the increase of $G / D$. When the small pipe contacts the main pipe, the drag force on the main pipe has the maximum value. With the increase of $G / D$, the effect of the small pipe becomes weak and the drag force on the main pipe decreases to the value for a single pipe. It is indicated that the existence of the smal pipe increases the total drag on the pipeline system, which is consistent with the conclusion by the published literature [17].

The mean value of $C_{L}$ increases with the increase of $G / D$ until it reaches its maximum value at $G / D \approx 0.25$. Then the mean value of $C_{L}$ decreases with the increasing $G / D$ and converges to the value for a single pipe. This can be explained by that the vortex shedding from the upper side of the main pipe is suppressed most effectively by the small pipe at $G / D \approx 0.25$ [2] and the clockwise vortices shed from the lower side of the cylinder lead to an upward (positive) lift force [27]. It is the main reason 


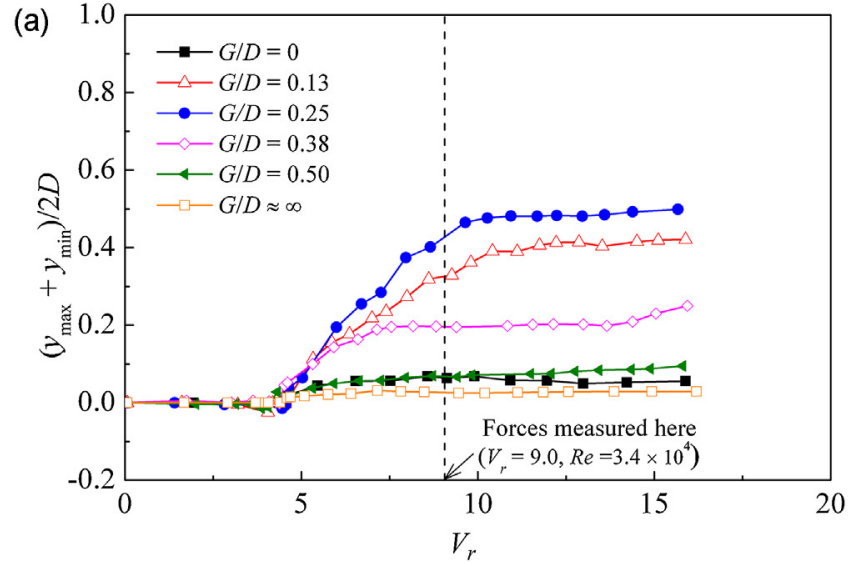

(b)

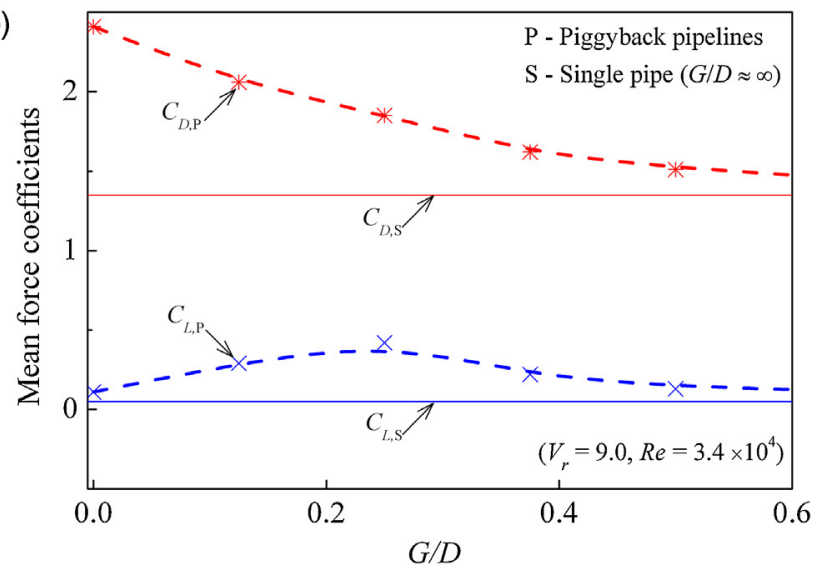

Fig. 7. (a) Mean position of vibrating piggyback pipelines for different spacingto-diameter ratios and (b) variation of mean force coefficients of main pipe with spacing-to-diameter ratio $\left(e / D=1.0, d / D=0.25, \theta=90^{\circ}, \alpha \approx 0.160, \operatorname{Re}=2.1-3.2 \times 10^{4}\right)$.

of the piggyback pipelines raised from the initial position during vibration, as shown in Fig. 7(a).

Fig. 8(a) shows the variation of the peak amplitude $(A / D)_{\max }$ with $G / D$. $(A / D)_{\max }$ decreases with the increase of $G / D$ when $G / D<0.25$ and reaches its minimum value at $G / D \approx 0.25$. Then $(A / D)_{\max }$ slightly increases and keeps constant thereafter with the increase of $G / D$. The Reynolds number at the peak amplitudes varies in a narrow range $\left(\left.\operatorname{Re}\right|_{(A / D)_{\max }}=2.0-2.6 \times 10^{4}\right)$, only except for $G / D=0.25$ $\left(\left.\operatorname{Re}\right|_{(A / D)_{\max }}=3.2 \times 10^{4}\right)$. According to Govardhan and Williamson [10], the peak amplitude increases with the increase of $\left.R e\right|_{(A / D)_{\max }}$ for a given $\alpha$. However, in this set of tests, the minimum peak amplitude occurs at the high Reynolds number, i.e. $G / D=0.25$. This is mainly because of the most significant suppression effect of the small pipeline at this value of $G / D$, even though the effect of Reynolds number has been considered. Fig. 8(a) also indicates that the amplitude of VIV would be enhanced if the small pipe is too close to the main pipe by comparison with the value for a single pipe.

Fig. 8(b) shows the variation of the critical reduced velocity $\left(V_{r, \text { onset }}\right)$ with the $G / D$. $V_{r, \text { onset }}$ increases with the increase of $G / D$ as $G / D<0.25$ and decreases as $G / D>0.25$. $V_{r, \text { onset }}$ at $G / D=0$ is smaller than that for a single pipe (solid line), indicating that the onset of VIV is more easily to occur than a single pipe when the small pipe is in contact with the main pipe. At $G / D=0.5$, the small pipe has little effect on the main pipe and the value of $V_{r, \text { onset }}$ is close to that for a single pipe. The maximum value of $V_{r, \text { onset }}$ occurs at $G / D \approx 0.25$. The results of both the peak amplitude and the critical reduced velocity indicate that VIV is suppressed most effectively by the small pipe at the spacing-to-diameter ratio of $G / D \approx 0.25$ for $\theta=90^{\circ}$.

\subsubsection{Effect of position angle $\theta$}

The position angle of the small pipe relative to the main pipe was changed from $0^{\circ}$ to $180^{\circ}$ to investigate its effect on the VIV response. The gap-to-diameter ratio is set at $G / D=0.25$; the diameter ratio is $d / D=0.25$ and the mass-damping parameter $\alpha \approx 0.160$.

The vibration amplitudes versus the reduced velocity for different position angles are shown in Fig. 9(a). It can be seen that the behavior of piggyback pipelines varies remarkably with the change in the position angle. For $\theta=0^{\circ}$, i.e. the small pipe is in front of the main pipe, the piggyback pipelines vibrate at high amplitudes and the right boundary of the lock-in regime is beyond the maximum reduced velocity that the test facility can generate. For $\theta=30^{\circ}$, the resonance only occurs in a narrow
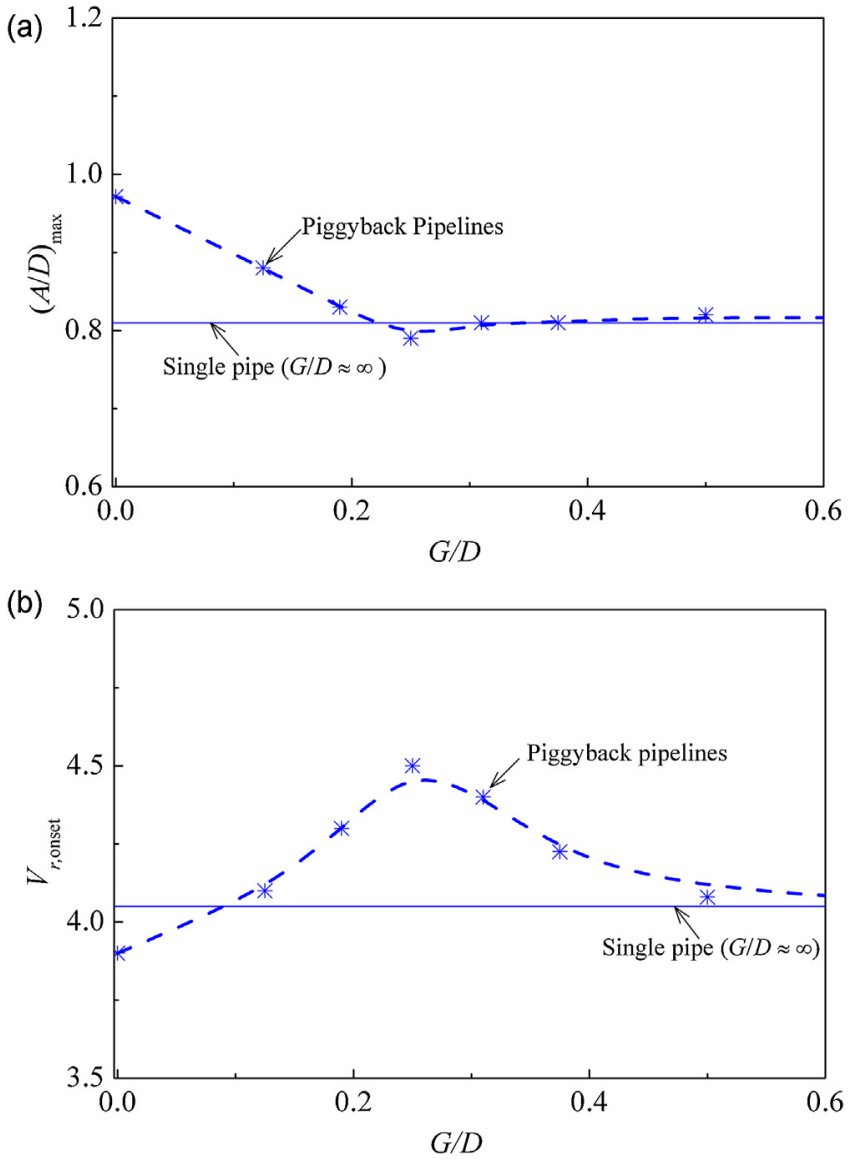

Fig. 8. (a) Variation of peak amplitude of VIV with spacing-to-diameter ratio and (b) variation of onset reduced velocity with spacing-to-diameter ratio ( $e / D=1.0$, $d / D=0.25, \theta=90^{\circ}, \alpha \approx 0.160, R e=2.1-3.2 \times 10^{4}$ ).
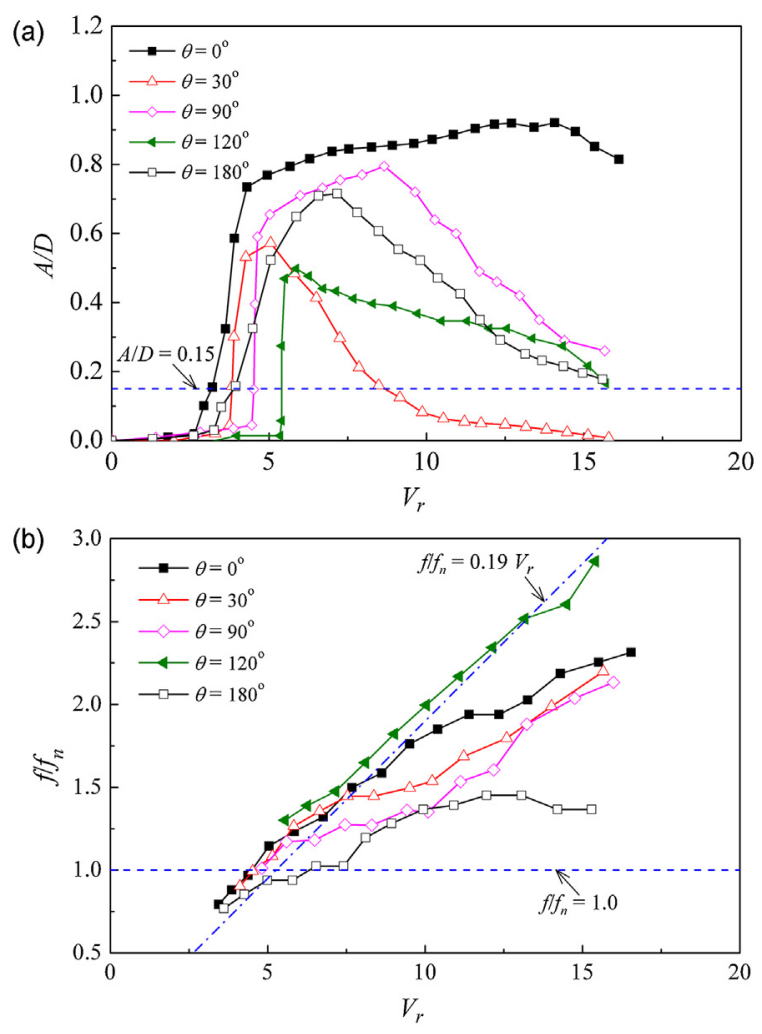

Fig. 9. VIV response of piggyback pipelines for different position angles: (a) amplitude and (b) frequency $\left(e / D=1.0, G / D=0.25, d / D=0.25, \alpha \approx 0.160, R e=1.8-4.6 \times 10^{4}\right)$. 


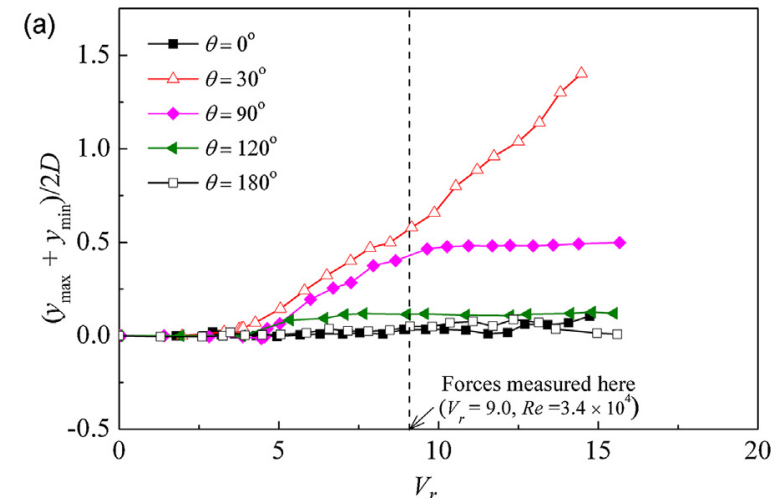

(b)

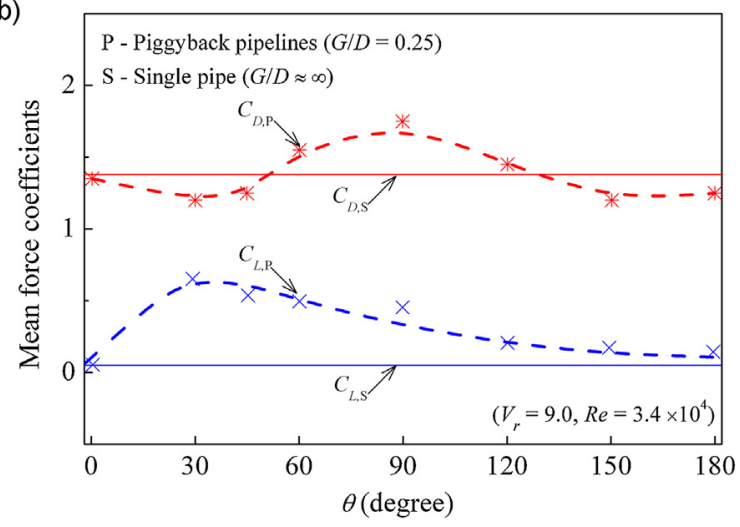

Fig. 10. (a) Mean position of vibrating piggyback pipelines for different position angles and (b) variation of mean force coefficients of main pipe with position angle $\left(e / D=1.0, G / D=0.25, d / D=0.25, \alpha \approx 0.160, R e=1.8-4.6 \times 10^{4}\right)$.

range of $V_{r}$ and has small peak amplitudes. For $\theta=120^{\circ}$, the onset of VIV occurs at a higher reduced velocity than at other position angles and the vibration amplitudes are kept at small values. The curve for $\theta=0^{\circ}$ has the highest amplitude and the widest lock-in range among these position angles. For $\theta=90^{\circ}$ and $180^{\circ}$, the vibration has the medium amplitudes and "lock-in" ranges.

The normalized frequency $\left(f / f_{n}\right)$ of VIV varying with the reduced velocity is shown in Fig. 9(b). $f / f_{n}$ generally increases with the increasing $V_{r}$. At the initial stage of VIV $\left(V_{r}<5\right), f / f_{n}$ follows the Strouhal law $\left(f / f_{n}=0.19 V_{r}\right)$, indicating that the vortex shedding dominates the vibration of the piggyback pipelines. As the reduced velocity exceeds 5.0, the position angle has significant effect on $f / f_{n} . f / f_{n}$ almost covers the area between the Strouhal line and the line of $f / f_{n}=1.0$ because of the complex interactions between the fluid and pipelines. For $\theta=120^{\circ}, f / f_{n}$ almost follows the Strouhal line in the lock-in regime and has the highest vibration frequency due to the absolute control by the vortex shedding. For $\theta=180^{\circ}$, the value of $f / f_{n}$ has the lowest vibrating frequency, which means that the vortex shedding is controlled by the vibrating structures, the inertia dominating the behavior of the piggyback pipelines.

Fig. 10(a) shows the mean positions of the vibration for different position angles. For the tandem configurations of the two pipes $\left(\theta=0^{\circ}\right.$ and $\left.180^{\circ}\right)$, the mean positions of vibration are very close to zero due to the symmetric configuration. For other position angles, all the mean positions are above the initial balance position, namely the whole pipeline system is raised by the flow, especially for $\theta=30^{\circ}$.

The variations of the mean drag and lift coefficients of the main pipe with $\theta$ at $V_{r}=9.0\left(R e=3.4 \times 10^{4}\right)$ are plotted in Fig. $10(\mathrm{~b})$. The mean drag coefficient has a larger value for $\theta$ ranging in $60-120^{\circ}$ than other position angles. This is likely because that the project of the pipeline system in the transverse direction is increased when the piggyback is installed above the main pipe $\left(60^{\circ} \leq \theta \leq 120^{\circ}\right)$. The blockage effect of the pipeline system on the flow is more significant than a single pipe. For $\theta<60^{\circ}$ or $\theta>120^{\circ}$, the project of the piggyback on the vertical plane is still within that of the main pipe, thus the blockage effect is not evident.

The mean lift coefficient has the maximum value at about $\theta=30^{\circ}$, which can well explain the maximum upward deflection of the pipeline system occurring at $\theta=30^{\circ}$ in Fig. 10(a). The smallest values of mean $C_{L}$ occur at the two tandem configurations, i.e. $\theta=0^{\circ}$ and $180^{\circ}$, which can be reflected by the lowest mean positions at these two angles. The mean lift forces for all the position angles are pointing upward due to the existence of the small pipe.

Fig. 11(a) shows the peak amplitude of VIV $(A / D)_{\max }$ varying with the position angle. It can be seen that there are three peak values of $(A / D)_{\max }$ occurring at $\theta \approx 0^{\circ}$, $60^{\circ}$ and $180^{\circ}$, respectively. The two trough values of $(A / D)_{\max }$ occur at $\theta \approx 30^{\circ}$ and
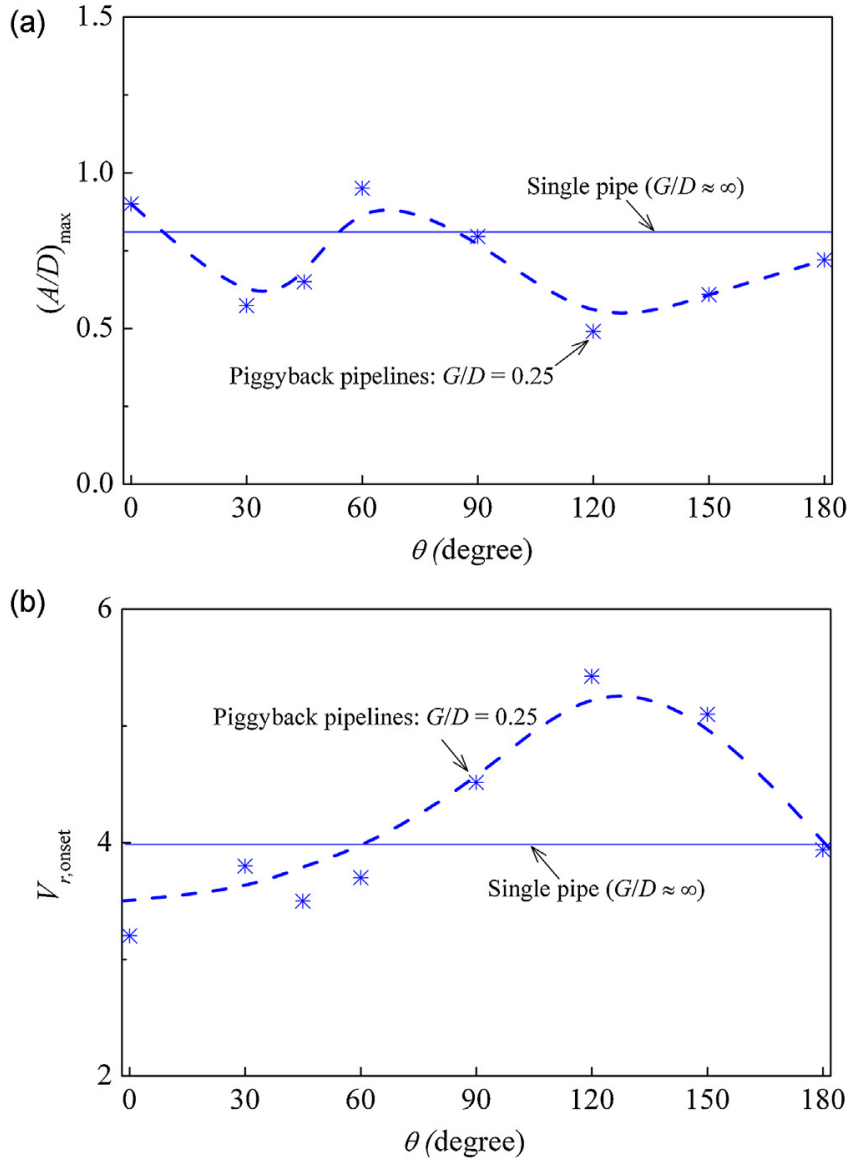

Fig. 11. (a) Variation of peak amplitude of VIV with position angles and (b) variation of onset reduced velocity with position angle $(e / D=1.0, G / D=0.25, d / D=0.25$, $\left.\alpha \approx 0.160, \operatorname{Re}=1.8-4.6 \times 10^{4}\right)$.

$120^{\circ}$. Rahmanian et al. [20] numerical results for the 2DOF VIV of piggyback pipelines at $V_{r}=8$ shown the peak values of $(A / D)_{\max }$ occur at $\theta=67.5^{\circ}$ and $150^{\circ}$, and the trough values of $(A / D)_{\max }$ occur at $22.5^{\circ}$ and $112.5^{\circ}$. The trends of $(A / D)_{\max }$ varying with $V_{r}$ in both studies are generally similar. The different between the two sets of results is due to the fact that the study of Rahmanian et al. [20] is for uniform flow past two cylinders, where there is no effects from the plane boundary. Apart of the influence from the gap in this study, two other reasons may also contribute to the difference between the two studies. One is the difference in diameter ratio and mass ratio; the other is that the VIV responses in Rahmanian et al. [20] were simulated at a constant reduced velocity, which may not represent the maximum amplitude in the whole lock-in regime. The Reynolds number of the peak amplitude for most cases varies from $1.8 \times 10^{4}$ to $2.6 \times 10^{4}$ and it may have slight influence on the value of the peak amplitude.

Fig. 11(b) shows the variation of the critical reduced velocity with the position angle. $V_{r, \text { onset }}$ for a single pipe is shown by a solid line for comparison. It can be seen that the position angle has great effect on $V_{r \text {,onset. }} V_{r \text {,onset }}$ generally increases with the increasing $\theta$ when $\theta<120^{\circ}$ and decreases when $\theta>120^{\circ}$. For $\theta>60^{\circ}$, the values of $V_{r, \text { onset }}$ are larger than that for a single pipe, indicating that the onset of VIV of piggyback pipelines can be shifted to be delayed for the existence of the small pipe when $\theta>60^{\circ}$. The maximum value of $V_{r, \text { onset }}$ occurs at $\theta \approx 120^{\circ}$.

\section{Conclusions}

Transverse VIV of piggyback pipelines under the action of steady current was physically modeled with a hydro-elastic facility in conjunction with a water flume. The effects of the configuration parameters, including the mass-damping parameter $(\alpha)$, the diameter ratio $(d / D)$, the gap-to-diameter ratio $(e / D)$, the spacing-todiameter ratio $(G / D)$ and the position angle $(\theta)$, on the VIV response of piggyback pipelines are investigated. The conclusions are drawn as follows:

(1) Comparison with the prediction with the modified Griffin plot by Govardhan and Williamson [10] shows that the peak vibration amplitude of near-wall piggyback pipelines is smaller than that for a wall-free single pipe by up to $25 \%$ in present study, which indicates that the presences of the secondary pipe and the bottom effectively suppress the vortex-induced vibration. 
(2) The boundary effect on the VIV response is obvious for the near-bed piggyback pipelines. The peak amplitude decreases with the decrease of $e / D$. The critical reduced velocity and the vibration frequency increase with the decrease of $e / D$.

(3) For the configuration of the small pipe above the main pipe $\left(\theta=90^{\circ}\right)$, the minimum peak amplitude and the maximum critical reduced velocity occur at $G / D \approx 0.25$, which indicates that VIV is suppressed significantly by the small pipe at this spacing-to-diameter ratio.

(4) For the constant value of $G / D=0.25$, the vibration amplitude is suppressed most effectively around $\theta=30^{\circ}$ and $120^{\circ}$. The critical reduced velocity for $\theta>60^{\circ}$ is generally larger than that for a single pipe and the maximum value occurs at $\theta \approx 120^{\circ}$.

(5) The existence of the small pipe can lead to an upward mean lift force on the vibrating pipeline system, resulting in the rise of the pipeline system. The maximum mean lift force and the maximum deflection of displacement occur at $G / D \approx 0.25$ for $\theta=90^{\circ}$, and occur at $\theta \approx 30^{\circ}$ for $G / D=0.25$.

\section{Acknowledgements}

This work is financially supported by National Natural Science Foundation of China (Grant Nos. 51109202; 11372319). The authors would like to acknowledge the technical support and data analysis from Mr. Jinsheng Cui during his postgraduate study at Institute of Mechanics CAS.

\section{References}

[1] Zhao M, Cheng L, Teng B. Numerical modeling of flow and hydrodynamic forces around a piggyback pipeline near the seabed. Journal of Waterway, Port, Coastal and Ocean Engineering 2007:133(4):286-95.

[2] Zang ZP, Gao FP, Cui JS. Physical modeling and swirling strength analysis of vortex shedding from near-bed piggyback pipelines. Applied Ocean Research 2013;40:50-9.

[3] Feng CC [Master's thesis] The measurement of vortex-induced effects in flow past stationary and oscillation circular and D-section cylinders. Vancouver, BC, Canada: University of British Columbia; 1968.

[4] Moe G, Wu ZJ. The lift force on a cylinder vibrating in a current. Journal of Offshore Mechanics and Arctic Engineering 1990;112:297-303.

[5] Khalak A, Williamson CHK. Motions, forces and mode transitions in vortexinduced vibrations at low mass-damping. Journal of Fluids and Structures 1999;13(7-8):813-51.

[6] Bearman PW. Vortex shedding from oscillating bluff bodies. Annual Review of Fluid Mechanics 1984;16:195-222.

[7] Sumer BM, Fredsøe J. Hydrodynamics around cylinder structures. Singapore: World Scientific Press; 2006

[8] Sarpkaya T. A critical review of the intrinsic nature of vortex-induced vibrations. Journal of Fluids and Structures 2004;19(4):389-447.

[9] Williamson CHK, Govardhan RN. Vortex-induced vibrations. Annual Review of Fluid Mechanics 2004;36:413-55
[10] Govardhan RN, Williamson CHK. Defining the 'modified Griffin plot' in vortex-induced vibration: revealing the effect of Reynolds number using controlled damping. Journal of Fluid Mechanics 2006;561: $147-80$.

[11] Klamo JT, Leonard A, Roshko A. The effects of damping on the amplitude and frequency response of a freely vibrating cylinder in cross-flow. Journal of Fluids and Structures 2006;22:845-56.

[12] Vandiver JK. Damping parameters for flow-induced vibration. Journal of Fluids and Structures 2012;35:105-19.

[13] Zdravkovich MM. Flow induced oscillations of two interfering circular cylinders. Journal of Sound and Vibration 1985;101:511-21.

[14] Huera-Huarte FJ, Gharib M. Flow-induced vibrations of a side-by-side arrangement of two flexible circular cylinders. Journal of Fluids and Structures 2011;27(3):354-66.

[15] Zhou Y, Wang ZJ, So RMC, Xu SJ, Jin W. Free vibrations of two sideby-side cylinders in a cross-flow. Journal of Fluid Mechanics 2001;443: 197-229.

[16] Assi GRS, Bearman PW, Meneghini JR. On the wake-induced vibration of tandem circular cylinders: the vortex interaction excitation mechanism. Journal of Fluid Mechanics 2010;661:365-401.

[17] Kamarudin $\mathrm{MH}$, Thiagarajan KP Czajko A. Analysis of current-induced forces on offshore pipeline bundles. In: Fifth International Conference on Computational Fluid Dynamics in the Process Industries, vol. 1. 2006. p. 1-6.

[18] Zhao M, Cheng L, Teng B, Liang DF. Numerical simulation of viscous flow past two circular cylinders of different diameters. Applied Ocean Research 2005;27(1):39-55

[19] Tsutsui T, Igarashi T, Kamemoto K. Interactive flow around two circular cylinders of different diameters at close proximity. Experiment and numerica analysis by vortex method. Journal of Wind Engineering and Industrial Aerodynamics 1997;69-71:279-91.

[20] Rahmanian M, Zhao M, Cheng L, Zhou TM. Two-degree-of-freedom vortexinduced vibration of two mechanically coupled cylinders of different diameters in steady current. Journal of Fluids and Structures 2012;35:133-59.

[21] Zhou TM, Lalli A. Experimental studies of vortex-induced vibrations on a bundled cylinder system. In: Proceedings of the Fifth International Conference on Asian and Pacific Coasts. 2009. p. 267-74.

[22] Det Norske Veritas. Free spanning pipelines. Recommended practice DNVRPF105; 2006.

[23] Gao FP, Yang B, Wu YX, Yan SM. Steady current induced seabed scour around a vibrating pipeline. Applied Ocean Research 2006;28(5):291-8.

[24] Achenbach E. Distribution of local pressure and skin friction around a circular cylinder in cross-flow up to $\operatorname{Re}=5 \times 10^{5}$. Journal of Fluid Mechanics 1968:34(4):625-39.

[25] Lei CW, Cheng L, Armfield SW, Kavanagh K. Vortex shedding suppression for flow over a circular cylinder near a plane boundary. Ocean Engineering 2000;27:1109-27.

[26] Yang B, Gao FP, Jeng DS, Wu YX. Experimental study of vortex-induced vibrations of a pipeline near an erodible sandy seabed. Ocean Engineering 2008;35(3-4):301-5.

[27] Jauvtis N, Williamson CHK. The effect of two degrees of freedom on vortexinduced vibration at low mass and damping. Journal of Fluid Mechanics 2004;509:23-62. 